ACta Univ. Sapientiae, Mathematica, 11, 1 (2019) 172-185

DOI: 10.2478/ausm-2019-0014

\title{
Multiplication semimodules
}

\section{Rafieh Razavi Nazari}

Faculty of Mathematics,

K. N. Toosi University of Technology,

Tehran, Iran

email: rrazavi@mail.kntu.ac.ir

\section{Shaban Ghalandarzadeh}

Faculty of Mathematics, K. N. Toosi University of Technology, Tehran, Iran

email: ghalandarzadeh@kntu.ac.ir

\begin{abstract}
Let $S$ be a semiring. An $S$-semimodule $M$ is called a multiplication semimodule if for each subsemimodule $N$ of $M$ there exists an ideal I of $S$ such that $N=$ IM. In this paper we investigate some properties of multiplication semimodules and generalize some results on multiplication modules to semimodules. We show that every multiplicatively cancellative multiplication semimodule is finitely generated and projective. Moreover, we characterize finitely generated cancellative multiplication $\mathrm{S}$-semimodules when $\mathrm{S}$ is a yoked semiring such that every maximal ideal of $S$ is subtractive.
\end{abstract}

\section{Introduction}

In this paper, we study multiplication semimodules and extend some results of [7] and [17] to semimodules over semirings. A semiring is a nonempty set $S$ together with two binary operations addition $(+)$ and multiplication $(\cdot)$ such that $(S,+)$ is a commutative monoid with identity element $0 ;(S,$.$) is a monoid$ with identity element $1 \neq 0 ; 0 a=0=a 0$ for all $a \in S ; a(b+c)=a b+a c$ and $(b+c) a=b a+c a$ for every $a, b, c \in S$. We say that $S$ is a commutative semiring if the monoid $(S,$.$) is commutative. In this paper we assume that$ all semirings are commutative. A nonempty subset $I$ of a semiring $S$ is called an ideal of $S$ if $a+b \in I$ and $s a \in I$ for all $a, b \in I$ and $s \in S$. A semiring 
$S$ is called yoked if for all $a, b \in S$, there exists an element $t$ of $S$ such that $a+t=b$ or $b+t=a$. An ideal $I$ of a semiring $S$ is subtractive if $a+b \in I$ and $b \in I$ imply that $a \in I$ for all $a, b \in S$. A semiring $S$ is local if it has a unique maximal ideal. A semiring is entire if $a b=0$ implies that $a=0$ or $\mathrm{b}=0$. An element $\mathrm{s}$ of a semiring $S$ is a unit if there exists an element $\mathrm{s}^{\prime}$ of $S$ such that $s s^{\prime}=1$. A semiring $S$ is called a semidomain if for any nonzero element $a$ of $S, a b=a c$ implies that $b=c$. An element $a$ of a semiring $S$ is called multiplicatively idempotent if $a^{2}=a$. The semiring $S$ is multiplicatively idempotent if every element of $S$ is multiplicatively idempotent.

Let $(M,+)$ be an additive abelian monoid with additive identity $0_{M}$. Then $M$ is called an $S$-semimodule if there exists a scalar multiplication $S \times M \rightarrow M$ denoted by $(\mathrm{s}, \mathrm{m}) \mapsto \mathrm{sm}$, such that $\left(\mathrm{ss}^{\prime}\right) \mathrm{m}=\mathrm{s}\left(\mathrm{s}^{\prime} \mathrm{m}\right) ; \mathrm{s}\left(\mathrm{m}+\mathrm{m}^{\prime}\right)=\mathrm{sm}+\mathrm{sm}^{\prime}$; $\left(s+s^{\prime}\right) m=s m+s^{\prime} m ; 1 m=m$ and $s 0_{M}=0_{M}=0 m$ for all $s, s^{\prime} \in S$ and all $\mathrm{m}, \mathrm{m}^{\prime} \in \mathrm{M}$. A subsemimodule $\mathrm{N}$ of a semimodule $\mathrm{M}$ is a nonempty subset of $M$ such that $m+n \in N$ and $s n \in N$ for all $m, n \in N$ and $s \in S$. If $N$ and $L$ are subsemimodules of $M$, we set $(N: L)=\{s \in S \mid s L \subseteq N\}$. It is clear that $(\mathrm{N}: \mathrm{L})$ is an ideal of $\mathrm{S}$.

Let $R$ be a ring. An R-module $M$ is a multiplication module if for each submodule $N$ of $M$ there exists an ideal I of $R$ such that $N=I M$ [2]. Multiplication semimodules are defined similarly. These semimodules have been studied by several authors(e.g. [5], [6], [18], [20]). It is known that invertible ideals of a ring $\mathrm{R}$ are multiplication R-modules. Invertible ideals of semirings has been studied in [8]. In this paper, in order to study the relations between invertible ideals of semirings and multiplication semimodules, we generalize some properties of multiplication modules to multiplication semimodules (cf. Theorems 2 and 12). In Section 2, we show that if $M$ is a multiplication $S$ semimodule and $P$ is a maximal ideal of $S$ such that $M \neq P M$, then $M_{P}$ is cyclic. In Section 3, we study multiplicatively cancellative(abbreviated as MC) multiplication semimodules. We show that MC multiplication semimodules are finitely generated and projective. In Section 4, we characterize finitely generated cancellative multiplication semimodules over yoked semirings with subtractive maximal ideals.

\section{Multiplication semimodule}

In this section we give some results of multiplication semimodules which are related to the corresponding results in multiplication modules. 
Definition 1 [6] Let $\mathrm{S}$ be a semiring and $\mathrm{M}$ an $\mathrm{S}$-semimodule. Then $\mathrm{M}$ is called a multiplication semimodule if for each subsemimodule $\mathrm{N}$ of $\mathrm{M}$ there exists an ideal $\mathrm{I}$ of $\mathrm{S}$ such that $\mathrm{N}=\mathrm{IM}$. In this case it is easy to prove that $\mathrm{N}=(\mathrm{N}: \mathrm{M}) \mathrm{M}$. For example, every cyclic $\mathrm{S}$-semimodule is a multiplication S-semimodule [20, Example 2].

Example 1 Let $\mathrm{S}$ be a multiplicatively idempotent semiring. Then every ideal of $\mathrm{S}$ is a multiplication $\mathrm{S}$-semimodule. Let $\mathrm{J}$ be an ideal of $\mathrm{S}$ and $\mathrm{I} \subseteq \mathrm{J}$. If $x \in \mathrm{I}$, then $\mathrm{x}=\mathrm{x}^{2} \in \mathrm{IJ}$. Therefore $\mathrm{I}=\mathrm{IJ}$ and hence $\mathrm{J}$ is a multiplication S-semimodule.

Let $\mathrm{M}$ and $\mathrm{N}$ be $\mathrm{S}$-semimodules and $\mathrm{f}: \mathrm{M} \rightarrow \mathrm{N}$ an $\mathrm{S}$-homomorphism. If $M^{\prime}$ is a subsemimodule of $M$ and $I$ is an ideal of $S$, then $f\left(\operatorname{IM}^{\prime}\right)=\operatorname{If}\left(M^{\prime}\right)$. Now suppose that $f$ is surjective and $N^{\prime}$ is a subsemimodule of $N$. Put $M^{\prime}=$ $\left\{m \in M \mid f(m) \in N^{\prime}\right\}$. Then $M^{\prime}$ is a subsemimodule of $M$ and $f\left(M^{\prime}\right)=N^{\prime}$. It is well-known that every homomorphic image of a multiplication module is a multiplication module (cf. [7] and [19, Note 1.4]). A similar result holds for multiplication semimodules.

Theorem 1 Let $\mathrm{S}$ be a semiring, $\mathrm{M}$ and $\mathrm{N}$ S-semimodules and $\mathrm{f}: \mathrm{M} \rightarrow \mathrm{N}$ a surjective $\mathrm{S}$-homomorphism. If $\mathrm{M}$ is a multiplication $\mathrm{S}$-semimodule, then $\mathrm{N}$ is a multiplication $\mathrm{S}$-semimodule.

Proof. Let $\mathrm{N}^{\prime}$ be a subsemimodule of $\mathrm{N}$. Then there exists a subsemimodule $M^{\prime}$ of $M$ such that $f\left(M^{\prime}\right)=N^{\prime}$. Since $M$ is a multiplication $S$-semimodule, there exists an ideal I of $S$ such that $M^{\prime}=I M$. Then $N^{\prime}=f\left(M^{\prime}\right)=f(I M)=$ If $(M)=I N$. Therefore $N$ is a multiplication $S$-semimodule.

Fractional and invertible ideals of semirings have been studied in [8]. We recall here some definitions and properties.

An element $s$ of a semiring $S$ is multiplicatively-cancellable (abbreviated as $M C)$, if $s b=s c$ implies $b=c$ for all $b, c \in S$. We denote the set of all $M C$ elements of $S$ by $M C(S)$. The total quotient semiring of $S$, denoted by $Q(S)$, is defined as the localization of $S$ at $M C(S)$. Then $Q(S)$ is an $S$-semimodule and $S$ can be regarded as a subsemimodule of $Q(S)$. For the concept of the localization in semiring theory, we refer to [10] and [11]. A subset I of $Q(S)$ is called a fractional ideal of $S$ if $I$ is a subsemimodule of $Q(S)$ and there exists an $M C$ element $d \in S$ such that $d I \subseteq S$. Note that every ideal of $S$ is a fractional ideal. The product of two fractional ideals is defined by $\mathrm{IJ}=$ $\left\{a_{1} b_{1}+\ldots+a_{n} b_{n} \mid a_{i} \in I, b_{i} \in J\right\}$. A fractional ideal $I$ of a semiring $S$ is called invertible if there exists a fractional ideal $\mathrm{J}$ of $\mathrm{S}$ such that $\mathrm{IJ}=\mathrm{S}$. 
Now we restate the following property of invertible ideals from $[8$, Theorem 1.3] (see also [13, Proposition 6.3]).

Theorem 2 Let $\mathrm{S}$ be a semiring. An ideal $\mathrm{I}$ of $\mathrm{S}$ is invertible iff it is a multiplication $\mathrm{S}$-semimodule which contains an $\mathrm{MC}$ element of $\mathrm{S}$.

Let $M$ be an $S$-semimodule and $P$ a maximal ideal of $S$. Then similar to [7], we define $T_{P}(M)=\{m \in M \mid$ there exist $s \in S$ and $q \in P$ such that $s+q=$ 1 and $s m=0$. Clearly $T_{p}(M)$ is a subsemimodule of $M$. We say that $M$ is $\mathrm{P}$-cyclic if there exist $\mathrm{m} \in \mathrm{M}, \mathrm{t} \in \mathrm{S}$ and $\mathrm{q} \in \mathrm{P}$ such that $\mathrm{t}+\mathrm{q}=1$ and $\mathrm{tM} \subseteq \mathrm{Sm}$.

The following two theorems can be thought of as a generalization of $[7$, Theorem 1.2] (see also [5, Proposition 3]).

Theorem 3 Let $\mathrm{M}$ be an $\mathrm{S}$-semimodule. If for every maximal ideal $\mathrm{P}$ of $\mathrm{S}$ either $\mathrm{T}_{\mathrm{P}}(\mathrm{M})=\mathrm{M}$ or $\mathrm{M}$ is $\mathrm{P}$-cyclic, then $\mathrm{M}$ is a multiplication semimodule.

Proof. Let $N$ be a subsemimodule of $M$ and $I=(N: M)$. Then $I M \subseteq N$. Let $x \in N$ and $J=\{s \in S \mid s x \in I M\}$. Clearly $J$ is an ideal of $S$. If $J \neq S$, then by $[9$, Proposition 6.59] there exists a maximal ideal $\mathrm{P}$ of $\mathrm{S}$ such that $\mathrm{J} \subseteq \mathrm{P}$. If $M=T_{P}(M)$, then there exist $s \in S$ and $q \in P$ such that $s+q=1$ and $s x=0 \in I M$. Hence $s \in J \subseteq P$ which is a contradiction. So the second case will happen. Therefore there exist $m \in M, t \in S$ and $q \in P$ such that $t+q=1$ and $\mathrm{tM} \subseteq \mathrm{Sm}$. Thus $\mathrm{tN}$ is a subsemimodule of $\mathrm{Sm}$ and $\mathrm{tN}=\mathrm{Km}$ where $\mathrm{K}=\{\mathrm{s} \in \mathrm{S} \mid \mathrm{sm} \in \mathrm{tN}\}$. Moreover, $\mathrm{tKM}=\mathrm{K} \mathrm{tM} \subseteq \mathrm{Km} \subseteq \mathrm{N}$. Therefore $\mathrm{tK} \subseteq \mathrm{I}$. Thus $\mathrm{t}^{2} x \in \mathrm{t}^{2} \mathrm{~N}=\mathrm{tKm} \subseteq \mathrm{IM}$. Hence $\mathrm{t}^{2} \in \mathrm{J} \subseteq \mathrm{P}$ which is a contradiction. Therefore $\mathrm{J}=\mathrm{S}$ and $\mathrm{x} \in \mathrm{IM}$.

Theorem 4 Suppose that $\mathrm{M}$ is an $\mathrm{S}$-semimodule. If $\mathrm{M}$ is a multiplication semimodule, then for every maximal ideal $\mathrm{P}$ of $\mathrm{S}$ either $\mathrm{M}=\{\mathrm{m} \in \mathrm{M} \mid \mathrm{m}=$ $\mathrm{qm}$ for some $\mathrm{q} \in \mathrm{P}\}$ or $\mathrm{M}$ is $\mathrm{P}$-cyclic.

Proof. Let $P$ be a maximal ideal of $S$ and $M=P M$. If $m \in M$, then there exists an ideal $\mathrm{I}$ of $\mathrm{S}$ such that $\mathrm{Sm}=\mathrm{IM}$. Hence $\mathrm{Sm}=\mathrm{IPM}=\mathrm{PIM}=\mathrm{Pm}$. Therefore $\mathrm{m}=\mathrm{q} \mathrm{m}$ for some $\mathrm{q} \in \mathrm{P}$. Now let $M \neq P M$. Thus there exists $x \in M$ such that $x \notin P M$. Then there exists ideal I of $S$ such that $S x=I M$.

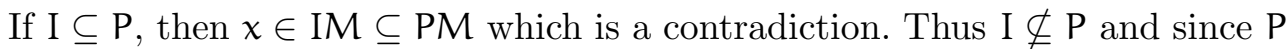
is a maximal ideal of $S, P+I=S$. Thus there exist $t \in I$ and $q \in P$ such that $\mathrm{q}+\mathrm{t}=1$. Moreover, $\mathrm{t} M \subseteq \mathrm{I} M=\mathrm{Sx}$. Therefore $M$ is P-cyclic.

We recall the following result from [10]. 
Theorem 5 A commutative semiring $\mathrm{S}$ is local iff for all $\mathrm{r}, \mathrm{s} \in \mathrm{S}, \mathrm{r}+\mathrm{s}=1$ implies $\mathrm{r}$ or $\mathrm{s}$ is a unit.

By using Theorem 4, we obtain the following corollary.

Corollary 1 Suppose that $(\mathrm{S}, \mathrm{m})$ is a local semiring. Let $\mathrm{M}$ be a multiplication $\mathrm{S}$-semimodule such that $\mathrm{M} \neq \mathrm{mM}$. Then $\mathrm{M}$ is a cyclic semimodule.

Proof. Since $M \neq m M, M$ is $m$-cyclic. Thus there exist $n \in M, t \in S$ and $\mathrm{q} \in \mathrm{m}$ such that $\mathrm{t}+\mathrm{q}=1$ and $\mathrm{tM} \subseteq \mathrm{Sn}$. Since $S$ is a local semiring, $\mathrm{t}$ is unit. Hence $M=S n$.

Remark 1 Let $\mathrm{S}$ be a semiring and $\mathrm{T}$ a non-empty multiplicatively closed subset of $\mathrm{S}$, and let $\mathrm{M}$ be an $\mathrm{S}$-semimodule. Define a relation $\sim$ on $\mathrm{M} \times \mathrm{T}$ as follows: $(\mathrm{m}, \mathrm{t}) \sim\left(\mathrm{m}^{\prime}, \mathrm{t}^{\prime}\right) \Longleftrightarrow \exists \mathrm{s} \in \mathrm{T}$ such that $\mathrm{stm}^{\prime}=\mathrm{st}^{\prime} \mathrm{m}$. The relation $\sim$ on $\mathrm{M} \times \mathrm{T}$ is an equivalence relation. Denote the set $\mathrm{M} \times \mathrm{T} / \sim$ by $\mathrm{T}^{-1} \mathrm{M}$ and the equivalence class of each pair $(\mathrm{m}, \mathrm{s}) \in \mathrm{M} \times \mathrm{T}$ by $\mathrm{m} / \mathrm{s}$. We can define addition on $\mathrm{T}^{-1} \mathrm{M}$ by $\mathrm{m} / \mathrm{t}+\mathrm{m}^{\prime} / \mathrm{t}^{\prime}=\left(\mathrm{t}^{\prime} \mathrm{m}+\mathrm{tm}^{\prime}\right) / \mathrm{tt}^{\prime}$. Then $\left(\mathrm{T}^{-1} \mathrm{M},+\right)$ is an abelian monoid. Let $\mathrm{s} / \mathrm{t} \in \mathrm{T}^{-1} \mathrm{~S}$ and $\mathrm{m} / \mathrm{u} \in \mathrm{T}^{-1} \mathrm{M}$. We can define the product of $\mathrm{s} / \mathrm{t}$ and $\mathrm{m} / \mathrm{u}$ by $(\mathrm{s} / \mathrm{t})(\mathrm{m} / \mathrm{u})=\mathrm{sm} / \mathrm{tu}$. Then it is easy to check that $\mathrm{T}^{-1} \mathrm{M}$ is an $\mathrm{T}^{-1} \mathrm{~S}$-semimodule [3]. Let $\mathrm{P}$ be a prime ideal in $\mathrm{S}$ and $\mathrm{T}=\mathrm{S} \backslash \mathrm{P}$. Then $\mathrm{T}^{-1} \mathrm{M}$ is denoted by $\mathrm{M}_{\mathrm{P}}$.

We can obtain the following results as in [15].

1. Suppose that $\mathrm{I}$ is an ideal of a semiring $\mathrm{S}$ and $\mathrm{M}$ is an $\mathrm{S}$-semimodule. Then $\mathrm{T}^{-1}(\mathrm{IM})=\mathrm{T}^{-1} \mathrm{IT}^{-1} \mathrm{M}$.

2. Let $\mathrm{N}, \mathrm{N}^{\prime}$ be subsemimodules of an $\mathrm{S}$-semimodule $\mathrm{M}$. If $\mathrm{N}_{\mathrm{m}}=\mathrm{N}_{\mathrm{m}}^{\prime}$ for every maximal ideal $\mathrm{m}$, then $\mathrm{N}=\mathrm{N}^{\prime}$.

Theorem 6 Let $\mathrm{S}$ be a semiring and $\mathrm{M}$ a multiplication $\mathrm{S}$-semimodule. If $\mathrm{P}$ is a maximal ideal of $\mathrm{S}$ such that $\mathrm{M} \neq \mathrm{PM}$, then $\mathrm{M}_{\mathrm{P}}$ is cyclic.

Proof. By (1), $M_{\mathrm{P}}$ is a multiplication $S_{\mathrm{P}}$-semimodule. Since $\mathrm{M} \neq \mathrm{PM}, \mathrm{M}_{\mathrm{P}} \neq$ $\mathrm{P}_{\mathrm{P}} M_{\mathrm{P}}$ by (2). Moreover, by [10, Theorem 4.5], $S_{\mathrm{P}}$ is a local semiring. Thus by Corollary $1, M_{P}$ is cyclic.

\section{MC multiplication semimodules}

In this section, we study MC multiplication semimodules and give some properties of these semimodules. 
In [4] an $S$-semimodule $M$ is called cancellative if for any $s, s^{\prime} \in S$ and $0 \neq \mathrm{m} \in M, \mathrm{sm}=\mathrm{s}^{\prime} \mathrm{m}$ implies $\mathrm{s}=\mathrm{s}^{\prime}$. We will call these semimodules multiplicatively cancellative(abbreviated as $M C$ ). For example every ideal of a semidomain $S$ is an MC S-semimodule.

Note that if $M$ is an $M C S$-semimodule, then $M$ is a faithful semimodule. Let $\mathrm{t} M=\{0\}$ for some $\mathrm{t} \in \mathrm{S}$. If $0 \neq \mathrm{m} \in M$, then $\mathrm{tm}=0 \mathrm{~m}=0$. Thus $\mathrm{t}=0$. Therefore $M$ is faithful. But the converse is not true. For example, if $S$ is an entire multiplicatively idempotent semiring, then every ideal of $S$ is a faithful $\mathrm{S}$-semimodule but it is not an MC semimodule.

Moreover, for an $R$-module $M$ over a domain $R, M$ is an $M C$ semimodule iff it is torsionfree. Also we know that if $R$ is a domain and $M$ a faithful multiplication R-module, then $M$ will be a torsionfree $R$-module and so $M$ is an MC semimodule.

An element $m$ of an $S$-semimodule $M$ is cancellable if $m+m_{1}=m+m_{2}$ implies that $m_{1}=m_{2}$. The semimodule $M$ is cancellative iff every element of $\mathrm{M}$ is cancellable [9, P. 172].

Lemma 1 Let $\mathrm{S}$ be a yoked entire semiring and $\mathrm{M}$ a cancellative faithful multiplication S-semimodule. Then $\mathrm{M}$ is an MC semimodule.

Proof. Let $0 \neq m \in M$ and $s, s^{\prime} \in S$ such that $s m=s^{\prime} m$. Since $S$ is a yoked semiring, there exists $t \in S$ such that $s+t=s^{\prime}$ or $s^{\prime}+t=s$. Suppose that $s+t=s^{\prime}$. Then $s m+t m=s^{\prime} m$. Since $M$ is a cancellative $S$-semimodule, $\mathrm{t} \mathrm{m}=0$. Moreover, there exists an ideal $\mathrm{I}$ of $\mathrm{S}$ such that $\mathrm{Sm}=\mathrm{IM}$ since $M$ is a multiplication $S$-semimodule. Then $\mathrm{tIM}=\mathrm{tSm}=\{0\}$ and hence $\mathrm{tI}=\{0\}$ since $M$ is faithful. But $S$ is an entire semiring, so $t=0$. Therefore $s=s^{\prime}$. Now suppose that $s^{\prime}+t=s$. A similar argument shows that $s=s^{\prime}$. Therefore $M$ is an $M C$ semimodule.

We now give the following definition similar to [12, P. 127].

Definition 2 Let $\mathrm{S}$ be a semidomain. An $\mathrm{S}$-semimodule $\mathrm{M}$ is said to be torsionfree if for any $0 \neq \mathrm{a} \in \mathrm{S}$, multiplication by $\mathrm{a}$ on $\mathrm{M}$ is injective, i.e., if $a x=$ ay for some $x, y \in M$, then $x=y$.

Theorem 7 Let $\mathrm{S}$ be a yoked semidomain and $\mathrm{M}$ a cancellative torsionfree $\mathrm{S}$-semimodule. Then $\mathrm{M}$ is an $\mathrm{MC}$ semimodule.

Proof. Let $0 \neq m \in M$ and $s, s^{\prime} \in S$ such that $s m=s^{\prime} m$. Since $S$ is a yoked semiring, there exists $t \in S$ such that $s+t=s^{\prime}$ or $s^{\prime}+t=s$. Suppose that $s+t=s^{\prime}$. Then $s m+t m=s^{\prime} m$. Since $M$ is a cancellative $S$-semimodule, 
$\mathrm{t} \mathrm{m}=0$. Since $M$ is a torsionfree $S$-semimodule, $\mathrm{m}=0$ which is a contradiction. Thus $t=0$ and hence $s=s^{\prime}$. Now suppose that $s^{\prime}+t=s$. A similar argument shows that $s=s^{\prime}$. Therefore $M$ is an $M C$ semimodule.

Now, similar to [7, Lemma 2.10] we give the following theorem (see also [6, Theorem 3.2]).

Theorem 8 Let $\mathrm{P}$ be a prime ideal of $\mathrm{S}$ and $\mathrm{M}$ an $\mathrm{MC}$ multiplication semimodule. Let $\mathrm{a} \in \mathrm{S}$ and $\mathrm{x} \in \mathrm{M}$ such that $\mathrm{ax} \in \mathrm{PM}$. Then $\mathrm{a} \in \mathrm{P}$ or $\mathrm{x} \in \mathrm{PM}$.

Proof. Let $a \notin P$ and put $K=\{s \in S \mid s x \in P M\}$. If $K \neq S$, there exists a maximal ideal $\mathrm{Q}$ of $\mathrm{S}$ such that $\mathrm{K} \subseteq \mathrm{Q}$. Let $\mathrm{M}=\mathrm{QM}$ and $\mathrm{m} \in \mathrm{M}$. Then similar to the proof of Theorem 4, there exists $\mathrm{q} \in \mathrm{Q}$ such that $\mathrm{m}=\mathrm{qm}$ which is a contradiction, since $M$ is an $M C$ semimodule. Therefore $M \neq Q M$. Thus by Theorem 4, we can conclude that $M$ is Q-cyclic. Therefore there exist $\mathrm{m} \in \mathrm{M}, \mathrm{t} \in \mathrm{S}$ and $\mathrm{q} \in \mathrm{Q}$ such that $\mathrm{t}+\mathrm{q}=1$ and $\mathrm{t} M \subseteq S \mathrm{~m}$. Thus $\mathrm{t} x=s \mathrm{~m}$ for some $s \in \mathrm{S}$. Moreover, $\mathrm{tPM} \subseteq \mathrm{Pm}$. Hence $\mathrm{tax} \in \mathrm{tPM} \subseteq \mathrm{Pm}$. Therefore $\operatorname{tax}=p_{1} m$ for some $p_{1} \in P$ and hence $a s m=p_{1} m$. Since $M$ is an $\mathrm{MC}$ semimodule, as $=\mathrm{p}_{1} \in \mathrm{P}$ and since $\mathrm{P}$ is a prime ideal, $\mathrm{s} \in \mathrm{P}$. Then $\mathrm{t} x=\mathrm{sm} \in \mathrm{PM}$ and hence $\mathrm{t} \in \mathrm{K} \subseteq \mathrm{Q}$ which is a contradiction. Thus $\mathrm{K}=\mathrm{S}$. Therefore $x \in P M$.

Lemma 2 (cf. [1]) Suppose that $\mathrm{S}$ is a semiring. Let $\mathrm{M}$ be an $\mathrm{S}$-semimodule and $\theta(\mathrm{M})=\sum_{\mathrm{m} \in \mathrm{M}}(\mathrm{Sm}: \mathrm{M})$. If $\mathrm{M}$ is a multiplication $\mathrm{S}$-semimodule, then $M=\theta(M) M$.

Proof. Suppose that $m \in M$. Then $S m=(S m: M) M$. Thus $m \in(S m: M)$ $M \subseteq \theta(M) M$. Therefore $M=\theta(M) M$.

Theorem 9 (cf. [7, Theorem 3.1]) Let $\mathrm{S}$ be a semiring and $\mathrm{M}$ an $\mathrm{MC}$ multiplication S-semimodule. Then the following statements hold:

1. If $\mathrm{I}$ and $\mathrm{J}$ are ideals of $\mathrm{S}$ such that $\mathrm{IM} \subseteq \mathrm{JM}$ then $\mathrm{I} \subseteq \mathrm{J}$.

2. For each subsemimodule $\mathrm{N}$ of $\mathrm{M}$ there exists a unique ideal $\mathrm{I}$ of $\mathrm{S}$ such that $\mathrm{N}=\mathrm{IM}$.

3. $\mathrm{M} \neq \mathrm{IM}$ for any proper ideal $\mathrm{I}$ of $\mathrm{S}$.

4. $\mathrm{M} \neq \mathrm{PM}$ for any maximal ideal $\mathrm{P}$ of $\mathrm{S}$.

5. $M$ is finitely generated. 
Proof. (1) Let IM $\subseteq J M$ and $a \in I$. Set $K=\{s \in S \mid s a \in J\}$. If $K \neq S$, there exists a maximal ideal $\mathrm{P}$ of $\mathrm{S}$ such that $\mathrm{K} \subseteq \mathrm{P}$. By Theorem $4, \mathrm{M}$ is $\mathrm{P}$-cyclic since $M$ is an $M C$ semimodule. Thus there exist $m \in M, t \in S$ and $q \in P$ such that $\mathrm{t}+\mathrm{q}=1$ and $\mathrm{t} M \subseteq \mathrm{Sm}$. Then $\mathrm{tam} \in \mathrm{tIM} \subseteq \mathrm{tJM}=\mathrm{Jt} M \subseteq \mathrm{Jm}$. Hence there exists $b \in J$ such that $t a m=b m$. Since $M$ is an $M C$ semimodule, $\mathrm{t} a=\mathrm{b} \in \mathrm{J}$. Thus $\mathrm{t} \in \mathrm{K} \subseteq \mathrm{P}$ which is a contradiction. Therefore $\mathrm{K}=\mathrm{S}$ and hence $\mathrm{I} \subseteq \mathrm{J}$.

(2) Follows by (1)

(3) Follows by (2)

(4) Follows by (3)

(5) By Lemma 2, $M=\theta(M) M$, where $\theta(M)=\sum_{m \in M}(S m: M)$. Then by $3, \theta(M)=S$. Thus there exist a positive integer $n$ and elements $m_{i} \in M$, $r_{i} \in\left(S m_{i}: M\right)$ such that $1=r_{1}+\ldots+r_{n}$. If $m \in M$, then $m=r_{1} m+\ldots+r_{n} m$. Therefore $M=S m_{1}+\ldots+S m_{n}$.

By Lemma 1, we have the following result.

Corollary 2 Let $\mathrm{S}$ be a yoked entire semiring and $\mathrm{M}$ a cancellative faithful multiplication S-semimodule. Then the following statements hold:

1. If $\mathrm{I}$ and $\mathrm{J}$ are ideals of $\mathrm{S}$ such that $\mathrm{IM} \subseteq \mathrm{JM}$ then $\mathrm{I} \subseteq \mathrm{J}$.

2. For each subsemimodule $\mathrm{N}$ of $\mathrm{M}$ there exists a unique ideal I of $\mathrm{S}$ such that $\mathrm{N}=\mathrm{IM}$.

3. $\mathrm{M} \neq \mathrm{IM}$ for any proper ideal $\mathrm{I}$ of $\mathrm{S}$.

4. $\mathrm{M} \neq \mathrm{PM}$ for any maximal ideal $\mathrm{P}$ of $\mathrm{S}$.

5. $M$ is finitely generated.

The concept of cancellation modules was introduced in [14]. Similarly we call an $S$-semimodule $M$ a cancellation semimodule if whenever IM $=\mathrm{JM}$ for ideals $\mathrm{I}$ and $\mathrm{J}$ of $\mathrm{S}$, then $\mathrm{I}=\mathrm{J}$.

Using the Theorem 9, we obtain the following corollary.

Corollary 3 Let $\mathrm{M}$ be an MC multiplication semimodule. Then $\mathrm{M}$ is a cancellation semimodule.

In [7, Lemma 4.1] it is shown that faithful multiplication modules are torsionfree. Similarly, we have the following result.

Theorem 10 Suppose that $\mathrm{S}$ is a semidomain and $\mathrm{M}$ is an MC multiplication $\mathrm{S}$-semimodule. Then $\mathrm{M}$ is a torsionfree $\mathrm{S}$-semimodule. 
Proof. Suppose that there exist $0 \neq t \in S$ and $m, m^{\prime} \in M$ such that $t \mathrm{~m}=\mathrm{tm}^{\prime}$. Then $S m=I M$ and $S m^{\prime}=J M$ for some ideals I, $J$ of $S$. Thus $t I M=t J M$ since $\mathrm{tm}=\mathrm{tm}^{\prime}$. By Corollary $3, \mathrm{M}$ is a cancellation semimodule, thus $\mathrm{tI}=\mathrm{t} J$. Let $x \in \mathrm{I}$. Then $\mathrm{t} x=\mathrm{t} x^{\prime}$ for some $x^{\prime} \in \mathrm{J}$. Since $S$ is a semidomain, $x=x^{\prime}$. Therefore $\mathrm{I} \subseteq \mathrm{J}$. Similarly $\mathrm{J} \subseteq \mathrm{I}$. Hence $\mathrm{I}=\mathrm{J}$ and $\mathrm{Sm}=\mathrm{Sm}^{\prime}$. Then there exists $s_{1} \in S$ such that $m=s_{1} m^{\prime}$. Thus $t m^{\prime}=t m=t s_{1} m^{\prime}$. Since $M$ is an MC semimodule, $t=s_{1} t$. Since $S$ is a semidomain, $s_{1}=1$. Therefore $m=m^{\prime}$ and hence $M$ is torsionfree.

If $M$ is a finitely generated faithful multiplication module, then $M$ is a projective module [17, Theorem 11]. Similarly, we have the following theorem:

Theorem 11 Let $\mathrm{M}$ be an MC multiplication semimodule. Then $\mathrm{M}$ is a projective S-semimodule.

Proof. By Theorem 9, $\theta(M)=\sum_{i}^{n}\left(S m_{i}: M\right)=S$. Thus for each $1 \leq i \leq n$, there exist $r_{i} \in\left(S m_{i}: M\right)$ and $s_{i} \in S$ such that $1=s_{1} r_{1}^{2}+\ldots+s_{n} r_{n}^{2}$. Define a map $\phi_{i}: M \rightarrow S$ by $\phi_{i}: m \mapsto s_{i} r_{i}$ a where $a$ is an element of $S$ such that $r_{i} m=a m_{i}$. Suppose that $a m_{i}=b m_{i}$ for some $b \in S$. Since $M$ is an $M C$ semimodule, $\mathrm{a}=\mathrm{b}$ and therefore $\phi_{i}$ is a well defined S-homomorphism. Let $m \in M$. Then $m=1 m=s_{1} r_{1}^{2} m+\ldots+s_{n} r_{n}^{2} m=\phi_{1}(m) m_{1}+\ldots+\phi_{n}(m) m_{n}$. By [16, Theorem 3.4.12], $M$ is a projective $S$-semimodule.

By Lemma 1, we obtain the following result.

Corollary 4 Let $\mathrm{S}$ be a yoked entire semiring and $\mathrm{M}$ a cancellative faithful multiplication $\mathrm{S}$-semimodule. Then $\mathrm{M}$ is a projective $\mathrm{S}$-semimodule.

Theorem 12 [7, Lemma 3.6] Let $\mathrm{S}$ be a semidomain and let $\mathrm{M}$ be an $\mathrm{MC}$ multiplication S-semimodule. Then there exists an invertible ideal I of S such that $\mathrm{M} \cong \mathrm{I}$.

Proof. Suppose that $0 \neq m \in M$. Then there exists an ideal $J$ of $S$ such that $\mathrm{Sm}=\mathrm{JM}$. Let $0 \neq \mathrm{a} \in \mathrm{J}$. We can define an $S$-homomorphism $\phi: M \rightarrow \mathrm{Sm}$ by $\phi: x \mapsto a x$. Let $x, x^{\prime} \in M$ such that $a x=a x^{\prime}$. By Theorem $10, M$ is torsionfree and hence $x=x^{\prime}$. Therefore $\phi$ is injective and so $M \cong f(M)$. Now define an $S$-homomorphism $\phi^{\prime}: S \rightarrow S m$ by $\phi^{\prime}(s)=$ sm. Let $s, s^{\prime} \in S$ such that $s m=s^{\prime} m$. Since $M$ is an MC semimodule, $s=s^{\prime}$. Therefore $\phi^{\prime}$ is injective. It is clear that $\phi^{\prime}$ is surjective. Therefore $S \cong S m$. Hence $M$ is isomorphic to an ideal $I$ of $S$. Thus I is a multiplication ideal and hence an invertible ideal of $S$. 


\section{Cancellative multiplication semimodule}

In this section, we investigate cancellative multiplication semimodules over some special semirings and restate some previous results. From now on, let $S$ be a yoked semiring such that every maximal ideal of $S$ is subtractive and let $M$ be a cancellative $S$-semimodule.

Theorem 13 (See Theorems 4 and 3) The S-semimodule $M$ is a multiplication $\mathrm{S}$-semimodule iff for every maximal ideal $\mathrm{P}$ of $\mathrm{S}$ either $\mathrm{M}$ is $\mathrm{P}$-cyclic or $M=\{m \in M \mid m=q m$ for some $q \in P\}$.

Proof. $(\Rightarrow)$ Follows by Theorem 4 .

$(\Leftarrow)$ Let $N$ be a subsemimodule of $M$ and $I=(N: M)$. Then $I M \subseteq N$. Let $x \in N$ and put $K=\{s \in S \mid s x \in I M\}$. If $K \neq S$, there exists a maximal ideal $P$ of $S$ such that $K \subseteq P$. If $M=\{m \in M \mid m=q m$ for some $q \in P\}$, then there exists $q \in P$ such that $x=q x$. Since $S$ is a yoked semiring, there exists $t \in S$ such that $t+1=q$ or $q+t=1$. Suppose that $q+t=1$. Then $q x+t x=x$ and hence $t x=0$. Therefore $t \in K \subseteq P$ which is a contradiction. Now suppose that $t+1=q$. Then $t x+x=q x$ and hence $t x=0$. Therefore $t \in K \subseteq P$. But $P$ is a subtractive ideal of $S$, so $1 \in P$ which is a contradiction. Therefore $M$ is $\mathrm{P}$-cyclic. Thus there exist $\mathrm{m} \in \mathrm{M}, \mathrm{t} \in \mathrm{S}$ and $\mathrm{q} \in \mathrm{P}$ such that $\mathrm{t}+\mathrm{q}=1$ and $t M \subseteq S m$. Therefore $t N$ is a subsemimodule of $S m$. Hence $t N=J m$ where $\mathrm{J}$ is the ideal $\{s \in S \mid s m \in t N\}$ of $S$. Then $t J M=J t M \subseteq J m \subseteq N$ and hence $\mathrm{t} J \subseteq \mathrm{I}$. Thus $\mathrm{t}^{2} x \in \mathrm{t}^{2} \mathrm{~N}=\mathrm{tJm} \subseteq \mathrm{IM}$. Therefore $\mathrm{t}^{2} \in \mathrm{K} \subseteq \mathrm{P}$ which is a contradiction.

Lemma 3 If $\mathrm{P}$ is a maximal ideal of $\mathrm{S}$, then $\mathrm{N}=\{\mathrm{m} \in \mathrm{M} \mid \mathrm{m}=\mathrm{qm}$ for some $\mathrm{q} \in \mathrm{P}\}$ is a subsemimodule of $\mathrm{M}$.

Proof. Let $\mathrm{m}_{1}, \mathrm{~m}_{2} \in \mathrm{N}$. Then there exist $\mathrm{q}_{1}, \mathrm{q}_{2} \in \mathrm{P}$ such that $\mathrm{m}_{1}=\mathrm{q}_{1} \mathrm{~m}_{1}$ and $\mathrm{m}_{2}=\mathrm{q}_{2} \mathrm{~m}_{2}$. Since $\mathrm{S}$ is a yoked semiring, there exits an element $\mathrm{r}$ such that $\mathrm{q}_{1}+\mathrm{q}_{2}+\mathrm{r}=\mathrm{q}_{1} \mathrm{q}_{2}$ or $\mathrm{q}_{1} \mathrm{q}_{2}+\mathrm{r}=\mathrm{q}_{1}+\mathrm{q}_{2}$. Since $\mathrm{P}$ is a subtractive ideal, $\mathrm{r} \in \mathrm{P}$.

Assume that $\mathrm{q}_{1} \mathrm{q}_{2}+\mathrm{r}=\mathrm{q}_{1}+\mathrm{q}_{2}$. Then $\mathrm{q}_{1} \mathrm{q}_{2}\left(\mathrm{~m}_{1}+\mathrm{m}_{2}\right)+\mathrm{r}\left(\mathrm{m}_{1}+\mathrm{m}_{2}\right)=$ $\left(q_{1}+q_{2}\right)\left(m_{1}+m_{2}\right)$. Thus $q_{1} q_{2} m_{1}+q_{1} q_{2} m_{2}+r\left(m_{1}+m_{2}\right)=q_{1} m_{1}+q_{2} m_{1}+$ $\mathrm{q}_{1} \mathrm{~m}_{2}+\mathrm{q}_{2} \mathrm{~m}_{2}$. Hence $\mathrm{q}_{2} \mathrm{~m}_{1}+\mathrm{q}_{1} \mathrm{~m}_{2}+\mathrm{r}\left(\mathrm{m}_{1}+\mathrm{m}_{2}\right)=\mathrm{q}_{1} \mathrm{~m}_{1}+\mathrm{q}_{2} \mathrm{~m}_{1}+\mathrm{q}_{1} \mathrm{~m}_{2}+\mathrm{q}_{2} \mathrm{~m}_{2}$. Since $M$ is a cancellative $S$-semimodule, $r\left(m_{1}+m_{2}\right)=q_{1} m_{1}+q_{2} m_{2}$. Thus $r\left(m_{1}+m_{2}\right)=m_{1}+m_{2}$. Therefore $m_{1}+m_{2} \in N$.

Now assume that $\mathrm{q}_{1}+\mathrm{q}_{2}+\mathrm{r}=\mathrm{q}_{1} \mathrm{q}_{2}$. Then $\left(\mathrm{q}_{1}+\mathrm{q}_{2}+\mathrm{r}\right)\left(\mathrm{m}_{1}+\mathrm{m}_{2}\right)=\mathrm{q}_{1} \mathrm{q}_{2}\left(\mathrm{~m}_{1}+\right.$ $\left.\mathrm{m}_{2}\right)$. Hence $\mathrm{q}_{1} \mathrm{~m}_{1}+\mathrm{q}_{1} \mathrm{~m}_{2}+\mathrm{q}_{2} \mathrm{~m}_{1}+\mathrm{q}_{2} \mathrm{~m}_{2}+\mathrm{r}\left(\mathrm{m}_{1}+\mathrm{m}_{2}\right)=\mathrm{q}_{1} \mathrm{q}_{2} \mathrm{~m}_{1}+\mathrm{q}_{1} \mathrm{q}_{2} \mathrm{~m}_{2}$. Thus $\mathrm{q}_{1} \mathrm{~m}_{1}+\mathrm{q}_{1} \mathrm{~m}_{2}+\mathrm{q}_{2} \mathrm{~m}_{1}+\mathrm{q}_{2} \mathrm{~m}_{2}+\mathrm{r}\left(\mathrm{m}_{1}+\mathrm{m}_{2}\right)=\mathrm{q}_{2} \mathrm{~m}_{1}+\mathrm{q}_{1} \mathrm{~m}_{2}$. Since $\mathrm{M}$ 
is a cancellative $\mathrm{S}$-semimodule, $\mathrm{q}_{1} \mathrm{~m}_{1}+\mathrm{q}_{2} \mathrm{~m}_{2}+\mathrm{r}\left(\mathrm{m}_{1}+\mathrm{m}_{2}\right)=0$ and hence $\mathrm{m}_{1}+\mathrm{m}_{2}+\mathrm{r}\left(\mathrm{m}_{1}+\mathrm{m}_{2}\right)=(1+\mathrm{r})\left(\mathrm{m}_{1}+\mathrm{m}_{2}\right)=0$. Since $\mathrm{P}$ is a subtractive ideal, $(1+\mathrm{r}) \notin \mathrm{P}$. Therefore $(1+\mathrm{r})+\mathrm{P}=\mathrm{S}$ since $\mathrm{P}$ is a maximal ideal of $\mathrm{S}$. Thus there exist $\mathrm{t} \in \mathrm{P}$ and $\mathrm{s} \in \mathrm{S}$ such that $\mathrm{s}(1+\mathrm{r})+\mathrm{t}=1$. Hence $\mathrm{s}(1+\mathrm{r})\left(\mathrm{m}_{1}+\mathrm{m}_{2}\right)+\mathrm{t}\left(\mathrm{m}_{1}+\mathrm{m}_{2}\right)=\mathrm{m}_{1}+\mathrm{m}_{2}$. Therefore $\mathrm{t}\left(\mathrm{m}_{1}+\mathrm{m}_{2}\right)=\mathrm{m}_{1}+\mathrm{m}_{2}$ and so $\mathrm{m}_{1}+\mathrm{m}_{2} \in \mathrm{N}$.

Let $\mathrm{s} \in \mathrm{S}$ and $\mathrm{m} \in \mathrm{N}$. Then there exists $\mathrm{q} \in \mathrm{P}$ such that $\mathrm{m}=\mathrm{qm}$. Thus $\mathrm{sm}=\mathrm{sqm}$. Since $\mathrm{sq} \in \mathrm{P}, \mathrm{sm} \in \mathrm{N}$. Therefore $\mathrm{N}$ is a subsemimodule of $\mathrm{M}$.

Similar to [7, Corollary 1.3], we have the following theorem.

Theorem 14 Let $M=\sum_{\lambda \in \Lambda} S_{m_{\lambda}}$. Then $M$ is a multiplication semimodule if and only if there exist ideals $\mathrm{I}_{\lambda}(\lambda \in \Lambda)$ of $\mathrm{S}$ such that $\mathrm{Sm}_{\lambda}=\mathrm{I}_{\lambda} M$ for all $\lambda \in \Lambda$.

Proof. $(\Rightarrow)$ Obvious.

$(\Leftarrow)$ Assume that there exist ideals $\mathrm{I}_{\lambda}(\lambda \in \Lambda)$ of $S$ such that $S \mathrm{~m}_{\lambda}=\mathrm{I}_{\lambda} \mathrm{M}(\lambda \in$ $\Lambda)$. Let $P$ be a maximal ideal of $S$ and $I_{\mu} \nsubseteq \subseteq$ for some $\mu \in \Lambda$. Then there exists $t \in I_{\mu}$ such that $t \notin P$. Thus $P+(t)=S$ and hence there exist $q \in P$ and $s \in S$ such that $1=q+$ st. Then $t s M \subseteq I_{\mu} M=S m_{\mu}$. Therefore $M$ is P-cyclic. Now suppose that $\mathrm{I}_{\lambda} \subseteq \mathrm{P}$ for all $\lambda \in \Lambda$. Then $\operatorname{Sm}_{\lambda} \subseteq \operatorname{PM}(\lambda \in \Lambda)$. This implies that $M=P M$. But for any $\lambda \in \Lambda, S m_{\lambda}=I_{\lambda} M=I_{\lambda} P M=P m_{\lambda}$. Therefore $\mathrm{m}_{\lambda} \in\{\mathrm{m} \in M \mid \mathrm{m}=\mathrm{qm}$ for some $\mathrm{q} \in \mathrm{P}\}$. Since by Lemma $3,\{\mathrm{~m} \in \mathrm{M} \mid \mathrm{m}=\mathrm{qm}$ for some $\mathrm{q} \in \mathrm{P}\}$ is an $\mathrm{S}$-semimodule, we conclude that $M=\{m \in M \mid m=q m$ for some $q \in P\}$. By Theorem $13, M$ is a multiplication semimodule.

It follows from Theorem 14 that if $S$ is a yoked semiring such that every maximal ideal of $S$ is subtractive, then any additively cancellative ideal I generated by idempotents is a multiplication ideal.

The following is a generalization of [7, Theorem 3.1]

Theorem 15 Let $\mathrm{M}$ be a faithful multiplication S-semimodule. Then the following statements are equivalent:

1. $M$ is finitely generated.

2. $\mathrm{M} \neq \mathrm{PM}$ for any maximal ideal $\mathrm{P}$ of $\mathrm{S}$.

3. If $\mathrm{I}$ and $\mathrm{J}$ are ideals of $\mathrm{S}$ such that $\mathrm{IM} \subseteq \mathrm{JM}$ then $\mathrm{I} \subseteq \mathrm{J}$.

4. For each subsemimodule $\mathrm{N}$ of $\mathrm{M}$ there exists a unique ideal I of $\mathrm{S}$ such that $\mathrm{N}=\mathrm{IM}$. 
5. $\mathrm{M} \neq \mathrm{IM}$ for any proper ideal $\mathrm{I}$ of $\mathrm{S}$.

Proof. (1) $\rightarrow$ (2) Let $P$ be a maximal ideal of $S$ such that $M=P M$ and $M=S m_{1}+\ldots+S m_{n}$. Since $M$ is a multiplication $S$-semimodule, for each $1 \leq i \leq n$, there exists $K_{i} \subseteq S$ such that $S m_{i}=K_{i} M=K_{i} P M=P K_{i} M=P m_{i}$. Therefore $m_{i}=p_{i} m_{i}$ for some $p_{i} \in P$. Since $S$ is a yoked semiring, there exists $t_{i} \in S$ such that $t_{i}+p_{i}=1$ or $1+t_{i}=p_{i}$. Suppose that $t_{i}+p_{i}=1$. Then $t_{i} m_{i}+p_{i} m_{i}=m_{i}$. Since $M$ is a cancellative $S$-semimodule, $t_{i} m_{i}=0$. Now suppose that $1+t_{i}=p_{i}$. Then $m_{i}+t_{i} m_{i}=p_{i} m_{i}$. Since $M$ is a cancellative $S$-semimodule, $t_{i} m_{i}=0$. Put $t=t_{1} \ldots t_{n}$. Then for all $i, t m_{i}=0$. Thus $t M=\{0\}$. Since $M$ is a faithful $S$-semimodule, $t=0 \in P$. Since $P$ is a prime ideal, $t_{i} \in P$ for some $1 \leq i \leq n$. If $t_{i}+p_{i}=1$, then $1 \in P$ which is a contradiction. If $1+t_{i}=p_{i}$, then, since $P$ is a subtractive ideal of $S, 1 \in P$ which is a contradiction. Therefore $M \neq P M$.

$(2) \rightarrow(3)$ Let I and J be ideals of $S$ such that $I M \subseteq J M$. Let $a \in I$ and put $K=\{r \in S \mid r a \in J\}$. If $K \neq S$, then there exists a maximal ideal $P$ of $\mathrm{S}$ such that $\mathrm{K} \subseteq \mathrm{P}$. By $2, \mathrm{M} \neq \mathrm{PM}$. Thus $\mathrm{M}$ is $\mathrm{P}$-cyclic and hence there exist $\mathrm{m} \in M, \mathrm{t} \in \mathrm{S}$ and $\mathrm{q} \in \mathrm{P}$ such that $\mathrm{t}+\mathrm{q}=1$ and $\mathrm{t} M \subseteq S \mathrm{~m}$. Then $\operatorname{tam} \in \mathrm{tJM}=\mathrm{Jt} M \subseteq J m$. Thus there exists $\mathrm{b} \in \mathrm{J}$ such that $\mathrm{tam}=\mathrm{bm}$. Since $S$ is a yoked semiring, there exists $c \in S$ such that $t a+c=b$ or $b+c=t a$. Suppose that $\mathrm{ta}+\mathrm{c}=\mathrm{b}$. Then $\mathrm{t}^{2} \mathrm{a}+\mathrm{tc}=\mathrm{tb}$ and $\mathrm{tam}+\mathrm{cm}=\mathrm{bm}$. Since $M$ is cancellative, $c m=0$. But $t c M \subseteq c(S m)=\{0\}$. Since $M$ is a faithful semimodule, $\mathrm{tc}=0$. Hence $\mathrm{t}^{2} \mathrm{a}=\mathrm{tb} \in \mathrm{J}$. Therefore $\mathrm{t}^{2} \in \mathrm{K} \subseteq \mathrm{P}$ which is a contradiction. Thus $S=K$ and $a \in J$. Now suppose that $b+c=t a$. Then $\mathrm{tb}+\mathrm{tc}=\mathrm{t}^{2} \mathrm{a}$ and $\mathrm{bm}+\mathrm{cm}=\mathrm{tam}$. Since $M$ is cancellative, $\mathrm{cm}=0$. A similar argument shows that $a \in J$.

(3) $\rightarrow$ (4) $\rightarrow$ (5) Obvious.

$(5) \rightarrow(1)$ By Lemma 2, $M=\theta(M) M$, where $\theta(M)=\sum_{m \in M}(S m: M)$. Then by $5, \theta(M)=S$. Thus there exist elements $m_{i} \in M, r_{i} \in\left(S m_{i}: M\right)$ such that $1=r_{1}+\ldots+r_{n}$. Now let $m \in M$. Then $m=r_{1} m+\ldots+r_{n} m$. Hence $M$ is finitely generated.

Theorem 8 can be restated as follows:

Theorem 16 (cf. [5, Proposition 3]) Suppose that $\mathrm{P}$ is a prime ideal and let $M$ be a faithful multiplication $S$-semimodule. Let $\mathrm{a} \in \mathrm{S}$ and $\mathrm{x} \in \mathrm{M}$ such that $\mathrm{a} x \in \mathrm{PM}$. Then $\mathrm{a} \in \mathrm{P}$ or $\mathrm{x} \in \mathrm{PM}$.

Proof. Let $a \notin P$ and $K=\{s \in S \mid s x \in P M\}$. Assume that $K \neq S$. Then there exists a maximal ideal $\mathrm{Q}$ of $\mathrm{S}$ such that $\mathrm{K} \subseteq \mathrm{Q}$. A similar argument to that of Theorem 13 shows that $\mathrm{M} \neq \mathrm{QM}$. Thus by Theorem 4, $\mathrm{M}$ is Qcyclic. Therefore there exist $\mathrm{m} \in M, \mathrm{t} \in \mathrm{S}$ and $\mathrm{q} \in \mathrm{Q}$ such that $\mathrm{t}+\mathrm{q}=1$ and 
$\mathrm{t} M \subseteq \mathrm{Sm}$. Thus $\mathrm{t} x=\mathrm{sm}$ for some $s \in \mathrm{S}$. Since $\mathrm{tPM} \subseteq \mathrm{Pm}, \mathrm{tax} \in \mathrm{tPM} \subseteq \mathrm{Pm}$. Hence $\operatorname{tax}=p_{1} m$ for some $p_{1} \in P$. Then asm $=p_{1} m$. Since $S$ is a yoked semiring, there exists $c \in S$ such that as $+c=p_{1}$ or $c+p_{1}=$ as. Suppose that $a s+c=p_{1}$. Then asm $+c m=p_{1} m$. Since $M$ is cancellative, $c m=0$. Then $\mathrm{tc} M \subseteq \mathrm{c}(\mathrm{Sm})=\{0\}$. Since $M$ is a faithful semimodule, $\mathrm{tc}=0$. Hence ast $=p_{1} t \in P$ and so $s \in P$ since $P$ is a prime ideal. Then $t x=s m \in P M$ and hence $\mathrm{t} \in \mathrm{K} \subseteq \mathrm{Q}$ which is a contradiction. Thus $\mathrm{K}=\mathrm{S}$. Therefore $\mathrm{x} \in \mathrm{PM}$. Now suppose that $c+p_{1}=a s$. A similar argument shows that $x \in P M$.

\section{References}

[1] D. D. Anderson, Y. Al-Shaniafi, Multiplication modules and the ideal ө(M), Comm. Algebra, 30 (7) (2002), 3383-3390.

[2] A. Barnard, Multiplication modules, J. Algebra, 71 (1981), 174-178.

[3] R. P. Deore, Characterizations of Semimodules, Southeast Asian Bull. Math., 36 (2012), 187-196.

[4] R. Ebrahimi Atani, S. Ebrahimi Atani, On subsemimodules of semimodules, Bul. Acad. Ştiinţe Repub. Mold. Mat., 63 (2) (2010), 20-30.

[5] S. Ebrahimi Atani, R. Ebrahimi Atani, U. Tekir, A Zariski topology for semimodules, Eur. J. Pure Appl. Math., 4 (3) (2011), 251-265.

[6] S. Ebrahimi Atani, M. Shajari Kohan, A note on finitely generated multiplication semimodules over commutative semirings, Int. J. Algebra, 4 (8) (2010), 389-396.

[7] Z. A. El-Bast, P. F. Smith, Multiplication modules, Comm. Algebra, 16 (4) (1988), 755-779.

[8] Sh. Ghalandarzadeh, P. Nasehpour and R. Razavi, Invertible ideals and Gaussian semirings, Arch. Math. (Brno), 53 (2017), 179-192.

[9] J. S. Golan, Semirings and Their Applications, Kluwer Academic Publishers, Dordrecht, 1999.

[10] C.B. Kim, A note on the localization in Semirings, J. Sci. Inst. Kook Min Univ, 3 (1985). 
[11] S. LaGrassa, Semirings: Ideals and Polynomials, PhD Thesis, University of Iowa, 1995.

[12] T. Y. Lam, Lectures on Modules and Rings, Springer-Verlag, 1998.

[13] M. D. Larsen, P. J. McCarthy, Multiplicative Theory of Ideals, Academic Press, New York, 1971.

[14] A. G. Naoum, A. S. Mijbass, Weak cancellation modules, Kyungpook Math. J., 37 (1997), 73-82.

[15] D. G. Northcott, Lessons on Rings, Modules and Multiplicity, New York: Cambridge University Press, 1968.

[16] M. Shabir, Some characterizations and sheaf representations of regular and weakly regular monoids and semirings, $\mathrm{PhD}$ Thesis, Quaid-I-Azam University, Pakistan, 1995.

[17] P. F. Smith, Some remarks on multiplication modules, Arch. Math. (Basel), 50 (1988), 223-235.

[18] H. A. Tavallaee, M. Zolfaghari, On semiprime subsemimodules and related results, J. Indones. Math. Soc., 19 (2013), 49-59.

[19] A. Tuganbaev, Multiplication modules, J. Math. Sci., 123 (2) (2004), 3839-3905.

[20] G. Yesilot, K. Orel, U. Tekir, On prime subsemimodules of semimodules, Int. J. Algebra, 4 (2010), 53-60. 